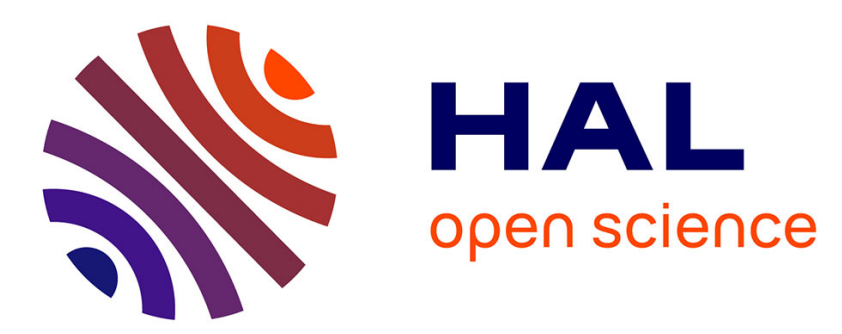

\title{
Multiphysics simulation to improve the understanding of pressure Wave Propagation techniques applied to composite polymers
}

\author{
Olivier Gallot-Lavallée, J.-L. Reboud, P. Rain
}

\section{- To cite this version:}

Olivier Gallot-Lavallée, J.-L. Reboud, P. Rain. Multiphysics simulation to improve the understanding of pressure Wave Propagation techniques applied to composite polymers. CEIDP, 2006, United States. pp.61-64. hal-00182611

\section{HAL Id: hal-00182611 https://hal.science/hal-00182611}

Submitted on 26 Oct 2007

HAL is a multi-disciplinary open access archive for the deposit and dissemination of scientific research documents, whether they are published or not. The documents may come from teaching and research institutions in France or abroad, or from public or private research centers.
L'archive ouverte pluridisciplinaire HAL, est destinée au dépôt et à la diffusion de documents scientifiques de niveau recherche, publiés ou non, émanant des établissements d'enseignement et de recherche français ou étrangers, des laboratoires publics ou privés. 


\title{
Multiphysics simulation to improve the understanding of Pressure Wave Propagation techniques applied to composite polymers
}

\author{
O. Gallot-lavallée, J.L. Reboud, P. Rain \\ LEMD, CNRS, Joseph Fourier University, Grenoble, France
}

\begin{abstract}
We used a model based on the finite element method. This method based on scattering of a structure by grid, enabled us to take into account the heterogeneity of the sample. The modelled structure is an electrical insulator sandwiched between two conductive electrodes. This configuration is similar to that used for the measurement of space charge by Pressure Wave Propagation. The sample is modelled by a visco-elastic matrix containing different fillers and space charges distributions. Specific electro mechanical boundary conditions were fixed. This led us to obtain dynamic results, such as the propagation of pressure wave in $2 \mathrm{~d}$ and the transient of electric displacement due to the principle of induced relative displacement. We selected the resulting ones that could help with the comprehension of the signals generated by these techniques.
\end{abstract}

\section{Introduction}

We propose the use of a numerical model to simulate the measurement of space charge by propagation of a pressure wave on composites polymers. Numerical simulations were performed with the SYSTUS ${ }^{\circledR}$ multiphysics software, developed by ESI Group. As first test case, we compared our simulation with the measurements obtained starting from a method based on relative displacement: the LASER induced pressure pulse method (LIPP Figure 1). This method is based on relative displacement; it consists in forcing the displacement of space charge compared to the electrodes creating by total influence a transient of charges in the electrodes (Figure 2). In practice, it's the luminous energy absorption by a target (for example a layer of Indian ink) deposited on the surface of the material which causes a brutal rise in temperature and thus a dilation of this layer creating a pressure wave on both sides. The slapping which one can hear is one of the consequences of this phenomenon, the other, being the creation of a longitudinal pressure wave being propagated towards the back of the target. Under certain conditions, in particular when the energy of the LASER is sufficiently large, the heating of the target is such as part of this one vaporizes; the associated matter ejection induced by reaction an additional compression. The Reference [1] contains a synthetic analysis of the guiding principles involved by this technique.

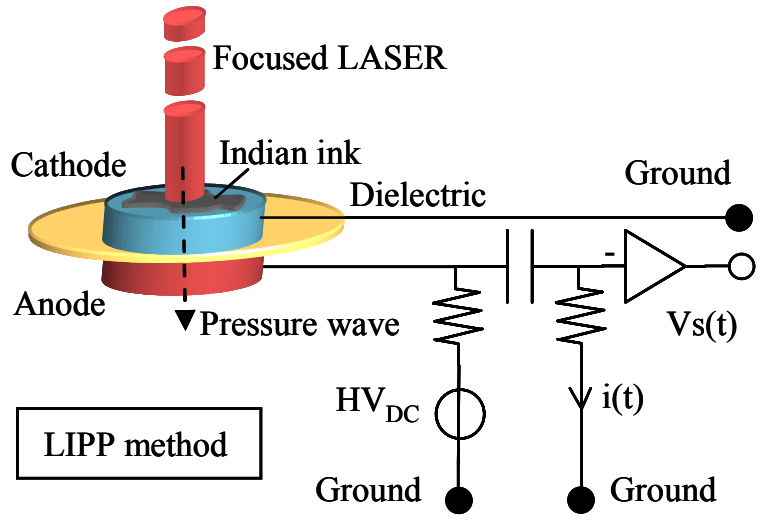

Figure 1: Functional diagram of the LIPP. The LASER causes a brutal dilation of the target of Indian ink, causing in its turn a normal pressure wave of to the top electrode. The wave is propagated through dielectric sandwiched by the electrodes. The local displacement of the space charges involves by total influence a fluctuation of influence charges. The conduction current representative of a distribution of space charge is then recorded via a high frequency reverser amplifier.

In this article, we describe the physical and geometrical model selected to represent this measurement technique, then we detail the methods of numerical resolution used. Three principal experiments are then declined. We confront in particular experiment and simulation on the basis of an epoxy sample subjected to a bias voltage cycle. Lastly, we simulate the influence of a heterogeneous structure (Epoxy - $\mathrm{SiO}_{2}$ particles) to such a measure.

\section{Model}

Geometry: Simulations are carried out on the basis of two-dimensional geometrical model (Figure 3) the model is however equipped with an additional dimension along $\mathrm{Z}$-axis in particular used during the surface calculation of charge density. The pressure wave is directly applied in entry of cathode. The substrate absorbing LASER beam is thus not modelled. The wave is propagated along $\mathrm{X}$-axis and crosses successively the cathode (K), the dielectric (D) and finally the anode (A). The mesh is regular within the three systems A, D and $\mathrm{K}$ and comprises only on cell along Y-axis. In the case of the composite dielectric, cutting in regular cells takes place in both direction $\mathrm{X}$ and $\mathrm{Y}$. Each cell is then individually comparable with a part of dielectric or 


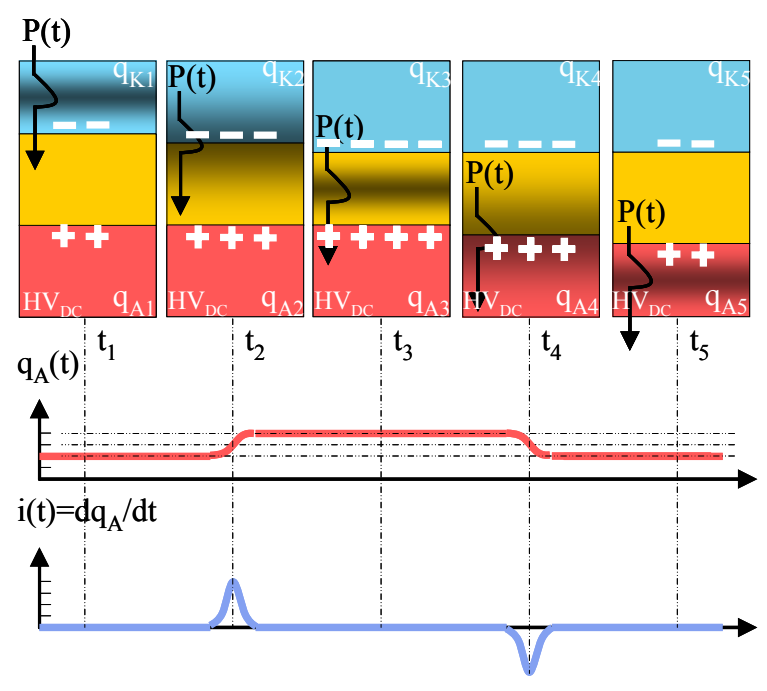

Figure 2: Simplified diagram of the measurement of space charge based on "the relative displacement". In top of this figure we represent the dielectric sandwiched by a cathode (K) and an anode (A) at the four moments characteristic of the wave propagation of pressure. In lower part we represent the influences charges of the anode according to time, like its derivative: the current of conduction. The analysis of this current makes it possible to reconstitute a charge distribution. By preoccupation with a simplification, we supposed the permittivity as a constant, the absence of dispersion and attenuation as well as a perfect adaptation of acoustic impedance.

with a particle starting from the tomography of the composite carried out by diffraction of x-ray (Figure 4).

Physics: The entry pressure wave is uniform along the $\mathrm{Y}$-axis transverse with the wave propagation. The boundary conditions are such as: the side edges are blocked according to $\mathrm{Y}$; the edge of exit is blocked according to $\mathrm{X}$ and $\mathrm{Y}$. The traditional visco-elastic properties are assigned to the dielectric, the electrodes and the particles in an isotropic way. It is considered that the topology reproduced a system in total influence. Coulomb strength, permittivity dependence and thermal are neglected, but might be taken into account in further simulations. The principal physical parameters of the model of simulation are pointed out (Table 1).

Numerical: The calculation of current is carried out according to the "Maxwell Ampere" equation simplified with the studied case i.e. magnetic induction B is neglected. All in all, the equation of the current density is governed by the following relation (1) where bold character stand for vector.

$$
\boldsymbol{J}=-\partial \mathbf{D} / \partial t
$$

Simulation allowing us to directly obtain the electric distribution of potential of the system during the

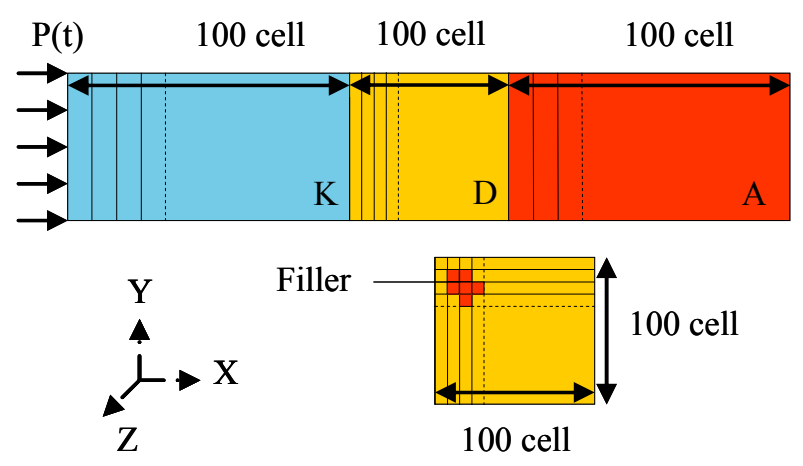

Figure 3: Two-dimensional model used for simulation. The pressure wave is propagated along $\mathrm{X}$-axis from cathode to anode while passing by the dielectric one. The meshing is regular. In the case of the composite a special meshing is carried out. The following boundary conditions are assumed: the plan of exit is blocked according to $\mathrm{X}$ and $\mathrm{Y}$ directions and the side plans are blocked according to $\mathrm{Y}$.

pressure wave propagation, we exploit (1) while revealing in (2) the field and the electrical current as well as the gain $\mathrm{G}$ of transformation of the current into voltage on the outlet side of the reverser amplifier. Calculates is carried out at the border between the dielectric one and the anode on the surface of contact $\mathrm{S}$.

$$
\begin{aligned}
& \boldsymbol{D}=\varepsilon_{0} \varepsilon_{r} \boldsymbol{E} \\
& \Rightarrow V S(t)=-\varepsilon_{0} \varepsilon_{r} G \int_{s}[\partial(\boldsymbol{E}) / \partial t] d s
\end{aligned}
$$

Numerical calculation (3) is thus based on the discretization of (2), where $\mathrm{H}$ represents the thickness of the system following $\mathrm{Z}$ making it possible to calculate the surface of contact and 1 the length (according to Y) of a basic cell. Calculation is carried out on each cell of index $\mathrm{i}$ located at the border of the interface insulatoranode.

$$
V S(t)=-\varepsilon_{0} \varepsilon_{r} G l H \sum_{i}\left[\boldsymbol{E}_{\mathbf{i}}(t)-\boldsymbol{E}_{\mathbf{i}}(t-1) / \Delta t\right]
$$

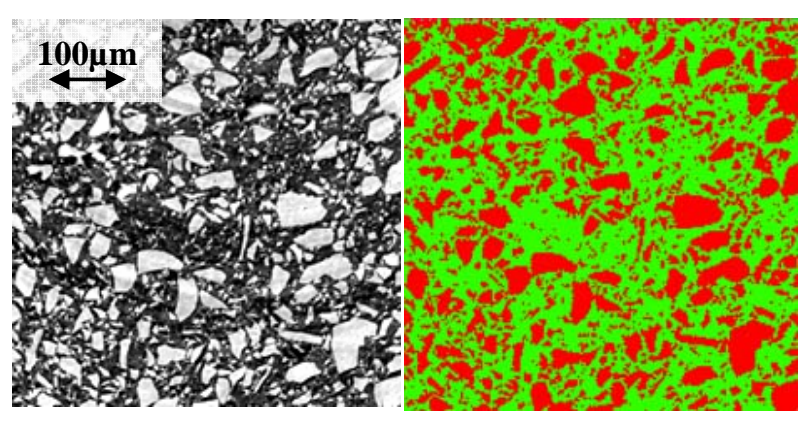

Figure 4: On the left here an X-ray imagery recorded by microtomography (from ESRF) in the middle of the epoxy sample filled with $\mathrm{SiO}_{2}(60 \%$ in weight). On the right here the discrete model of this object with the direct correspondence: 1 cell per pixel. The dark zones are comparable as $\mathrm{SiO}_{2}$. 
Table 1: Physical model

\begin{tabular}{ll}
\hline Physics & Parameters \\
\hline Coulomb strength & No \\
Configuration & Planar (total influence) \\
Relative permittivity & 3.5 (constant) \\
Young modulus & $7 \times 10^{9} \mathrm{MPa}$ (dielectric) \\
& $75 \times 10^{9} \mathrm{MPa}$ (electrodes) \\
& $95 \times 10^{9} \mathrm{MPa}$ (particles) \\
Density & $1200 \mathrm{Kg} / \mathrm{m}^{3}$ (dielectric) \\
& $2300 \mathrm{Kg} / \mathrm{m}^{3}($ electrodes) \\
& $2650 \mathrm{Kg} / \mathrm{m}^{3}$ (particles) \\
Dampening & $\alpha\left(\right.$ rigidity) $=0.5 \times 10^{-9} \mathrm{~s}^{-1}($ dielectric) \\
& $\beta(\mathrm{mass})=0.5 \times 10^{6} \mathrm{~s}($ dielectric) \\
Poisson coefficient & $0.3($ dielectric) \\
& $0.27(\mathrm{electrodes})$ \\
& $0.25(\mathrm{particles})$ \\
Deepness & $10 \mathrm{~mm}(\mathrm{H})$ \\
Isotropic & Yes \\
Wave shape & Gaussian \\
Wave amplitude & $0-30 \mathrm{MPa}$ (incidence versus X) \\
Wave passage time & $10 \mathrm{~ns}(1 / 2$ amplitude) \\
Wave pressure & Uniform (incidence versus Y) \\
Liberty degrees & Exit plan: $\mathrm{X}=0$ or $\mathrm{P}=0 / \mathrm{Y}=0$ \\
& Lateral plan: Y=0 \\
Thermal Dilatation & No \\
\hline
\end{tabular}

\section{Results}

Reflection's Modelling: We present (Figure 5) the electric response recorded on a sample of epoxy of $0.5 \mathrm{~mm}$ thickness moulded between two electrodes of $3.8 \mathrm{~mm}$ aluminium. The first peak observed corresponds in the passing of the pressure wave of $\mathrm{K}$ to $\mathrm{D}$ and the second corresponds in the passing of the pressure wave of $\mathrm{D}$ to A. Later one can identify a counterpart of these two peaks with however a quite different amplitude ratio, we will discuss this point below. The various peaks observed result from secondary pressures waves. Indeed to each passage from one medium to another, the wave gives raise to a component of reflection $R$ and transmission $T$, in accordance with relations (4) that govern amplitude ratio of $\mathrm{R}$ and $\mathrm{T}$ at the time of the passage of a medium $I$ in a medium II, of respective acoustic impedance $Z_{I}$ and $Z_{I I}$.

$$
R=\left(Z_{I I}-Z_{I}\right) /\left(Z_{I}+Z_{I I}\right) \text { and } T=2 Z_{I} /\left(Z_{I}+Z_{I I}\right)
$$

Measurements of space charges use only the first crossing of insulator. Thus, if measurements should not be disturbed, the duration of the two ways wave propagation in electrode $\mathrm{K}$ must be higher than the duration of the crossing of insulator.

The simulation of this measurement is presented (Figure 5). The physical model of this simulation was described in a previous paragraph. The parameters of adjustment of our simulation were the calibration of the pressure wave (amplitude and passage time) and the definition of the parameters of viscous damping. To simulate this experiment, we maintained the pressure equal to zero at the exit boundary in order to reproduce the phenomenon of reflection which takes place at the exit surface of $\mathrm{A}$ and $\mathrm{K}$ approximated to the vacuum.

The counterpart of the first two peaks - observed between $\mathrm{t}=1.86 \mu \mathrm{s}$ and $\mathrm{t}=2.1 \mu \mathrm{s}$ - is particularly interesting to explain. Figure 5 we represented the various routes of pressure wave caused by this technique. One understands thus why the peak corresponding to the passage of the anode is more important on the level of the counterpart, because at the same moment $\mathrm{t}=2 \mu \mathrm{s}$ two pressure waves come to combine their displacement effort. These waves which have followed different ways and which incidental according to opposed directions, have indeed undergoes a number of inversion respectively even $(=2)$ and odd $(=1)$, which allows this conjugation of effort. These behaviours are as well visible in simulation as in experimentation. The whole as of these observations confirm the good setting in mechanical parameter of the model.

Modelling with space charge: We took two successive measurements (Figure 6) on an epoxy resin in the first moments of polarization, then to resulting from $2 \mathrm{~h} 30$ of polarization. The experiment was carried out under a controlled atmosphere of $50^{\circ} \mathrm{C}$ with an aim of injecting homocharges on the two sides of the dielectric layer. On the stage of simulation, we sought to define a charge distribution in insulator so as to find a relative similarity with the experimentation. One shows as well as the charge distribution can be obtained in a completely reversed way. Indeed this new methodology consists in finding the charge distribution that gives a simulated electric answer nearest to that measured.

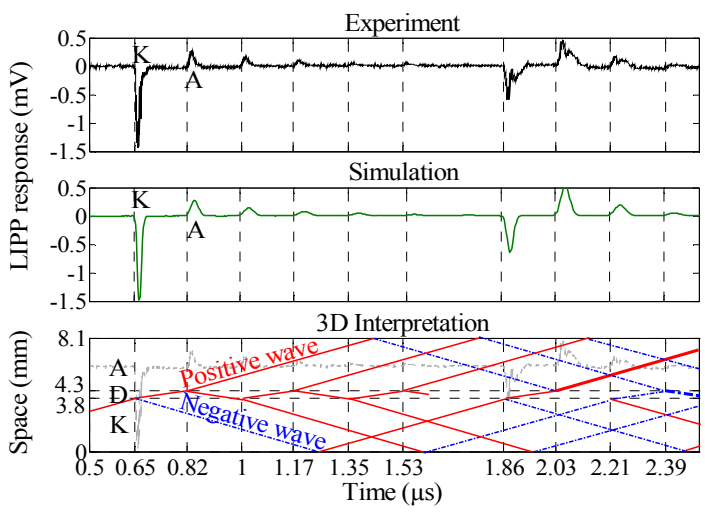

Figure 5: In the top figure we reproduced the electric response recorded with the LIPP on a sample of $0.5 \mathrm{~mm}$ thickness sandwiched between two electrodes of $3.8 \mathrm{~mm}$ thickness and polarized to $1 \mathrm{KV}$. The simulation of this measurement is reproduced in the central figure. Lastly, bellow of figure, we propose a representation (time, depth, pressure) of the advance of the principal wave and secondary waves. The acoustic impedance of $D$ is $Z d=2.9 \times 10^{\wedge} 6 \mathrm{~kg} / \mathrm{m}^{\wedge} 2 . \mathrm{s}, A$ and $\mathrm{K}$ is $\mathrm{Ze}=15 \times 10^{\wedge} 6 \mathrm{~kg} / \mathrm{m}^{\wedge} 2 . \mathrm{s}$ and the surrounding vacuum is $\mathrm{Zv}=0$. 
Lastly, on the side of simulation, it is interesting to see how the peak of exit corresponding to the test under polarization with space charge is shifted with respect to the peak under pure polarization. One thus should not forget to conceive that the pressure wave cannot be connected in true Dirac, this is why it is delicate to define exactly in which moment the wave of pressure crosses the border electrode-insulator dice at the time which we are in the presence of space charge.

Modelling with fillers: Figure 6, we simulated the electric response of an epoxy resin composite on the basis of distribution of silica particle imagery (Figure 4). We however took account only of mechanical heterogeneities of this new system excluding for the time being any electric heterogeneity. Thus this result of simulation makes it possible to imagine the impact of such a distribution on the pressure wave propagation. In accordance with what was expected, the wave propagates more quickly and undergoes a greater dispersion. Finally, the peak amplitude of entry is weakened with respect to the new impedance ratio. Indeed the total rigidity of materials increased with addition of particles thus limiting the electric displacement.

In practice the $\mathrm{SiO}_{2}$ grain reinforces the global permittivity, and we observe with final, appreciable increase in this peak since the apparent capacity is increased. Let us note overall that the existence of such a geometry and density of filler does not seem to be an important factor of disturbance of electric answer.

\section{Conclusions}

This work of simulations made it possible to find the principal signatures of this technique on the basis of extremely simplified model. We illustrated at which

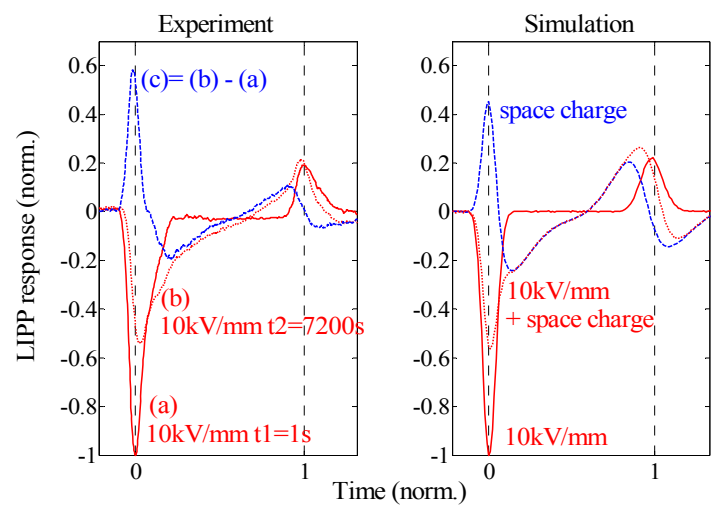

Figure 6: On the left stand the electric response measured after two different durations of polarization under $50^{\circ} \mathrm{C}$. We affixed this figure on the right corresponding simulation by seeking the space charge distribution which allowing this good similarity.

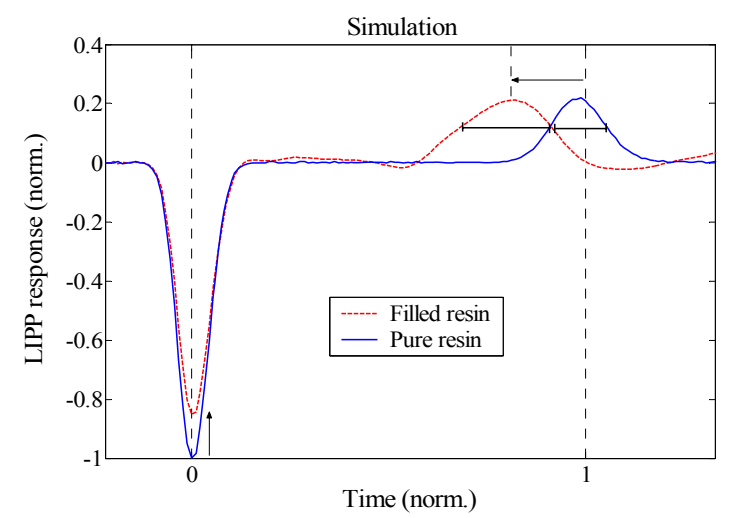

Figure 7: Here represented the simulation of an electric response of the LIPP on the basis of a pure sample and a composite (dashed line).

point it could be delicate to define the moment when the pressure wave passes from dielectric to the electrode. Thanks to simulation we could dissociate the effect of a mechanical heterogeneity of material on the wave propagation. Finally we proposed an alternative to the traditional exploitation of the LIPP, based on the random approach of the space charge distribution by fitting with the electric answers.

Beyond these first stages associating simulation and experimentation, we wish to facilitate the evaluation by simulation of certain constraints inaccessible to the experiment. The model must be used in the long run as support of comprehension of the interactions between various physical requests. It must in particular be able to be exploited with an aim of characterizing the ageing of dielectric materials and of proposing an evaluation of their reliability under conditions of given constraints. We are interested particularly in the constraints suitable for Electronics of Power: high tensions and electric frequencies $(\mathrm{kV} / \mu \mathrm{s})$, important temperatures and strong mechanical stresses.

\section{Reference}

[1] R. Gerhard-Multhaupt, "Analysis of pressure-wave methods for the nondestructive determination of spatial charge or field distributions in dielectrics", Physical Review B, vol. 27, pp. 2494-2503, Feb. 1983.

Author address: Olivier Gallot-lavallée, Associate Professor, LEMD-UJF, 25 Avenue des Martyrs, 38042 Grenoble, France, +33 (0)4.76.88.90,

Email: olivier.gallot-lavallee@grenoble.cnrs.fr

Acknowledgments : Tanks are due to ESI Group for providing SYSTUS ${ }^{\circledR}$ Multiphysics Software; G. Bertrand and G. Kermouche from ENI St Etienne for preliminary work and assistance. 